\title{
Designing Applications with Predictable Runtime Characteristics for the Baremetal Intel SCC
}

\author{
Devendra Rai, Lars Schor, Nikolay Stoimenov, \\ Iuliana Bacivarov, and Lothar Thiele \\ Computer Engineering and Networks Laboratory, \\ ETH Zurich, 8092 Zurich, Switzerland \\ firstname.lastname@tik.ee.ethz.ch
}

\begin{abstract}
Thus far, most of the research into many- core computation has focussed on improving the average performance of an application, such as the average throughput. However, applications such as medical ultrasound not only require high throughput, but also require predictable performance characteristics, and are generally implemented on platforms explicitly designed for real-time computing. This paper proposes design strategies which enable high performance multiprocessing applications to achieve predictable communication latencies, computation times and reaction latencies over on-chip many-core platforms, such as the Intel SCC. The strategies presented in this paper can be incorporated into existing many core application design frameworks, wherein the responsibility of achieving predictable timing performance can be relegated to the framework itself. Experiments using two representative applications validate our strategies and show that it is indeed possible to use on-chip many core platforms for real time multiprocessing.
\end{abstract}

Keywords: Multi-Processor System, On-Chip Many-Core System, Real Time, Single-chip Cloud Computer.

\section{Introduction}

Research in high performance computing has focussed primarily on improving the average performance (e.g., throughput, latency) of large applications. However, soft real time applications, such as the diagnostic medical ultrasound not only require high throughput, but also require that the application's run-time characteristics are consistent and predictable. Systems like the Intel SCC are viewed primarily as high performance computing platforms, rather than as platforms capable of providing predictable runtime environment. We believe that on-chip many-core platforms such as the Intel SCC will become mainstream in the near future. Other examples of such platforms are the Tilera and Kalray MPPA many core processors, see [1,2]. Therefore, it is interesting to ask whether it is possible to architect many-core applications which can execute with predictable performance characteristics on the Intel SCC, making such platforms

D. an Mey et al. (Eds.): Euro-Par 2013 Workshops, LNCS 8374, pp. 779-789, 2014.

○ Springer-Verlag Berlin Heidelberg 2014 
attractive for implementing soft real time high throughput applications. Moreover, it may be required that a given multiprocessing application react to events (e.g., control messages) in a bounded time. With this motivation, this paper proposes software design architecture with the following objectives:

1. Enable an application to execute with a predictable timing performance;

2. Enable an application to react to control messages in a predictable timeframe (henceforth, bounded reaction latency).

Our approach does not assume any special operating system features or services, and is therefore portable across platforms. We assume a general data-flow process network model of computation, which is extensively used to design and implement streaming applications(e.g., medical ultrasound). All results are validated on the baremetal SCC, and we emphasize that merely using the baremetal approach does not automatically lead to predicable performance characteristics.

\section{Related Work}

Although it is possible to port one of several multiprocessor real-time operating systems (RTOS) to the Intel SCC, an RTOS may consume a significant portion of the available computing resources on the chip, thus motivating the need for a baremetal solution, see [12. As a result, C-language based programming frameworks such as BareMetalC [7] and BareMichael [11] are now available which allow the applications to run directly on the SCC cores, without the need of an operating system. In addition, baremetal-compatible communication libraries such as RCCE [9], iRCCE [3] provide intra- and inter core communication. Puffitsch et. al. use the baremetal approach to execute task-sets in real time on the SCC, but their approach is restricted to periodic tasks with no pre-emption, and the tasks are not required to respond to events within a bounded latency, see [8]. In contrast, this paper assumes general data-flow process networks, which can react to (also, pre-empted by) events within a statically bounded time.

\section{Background}

\subsection{The Intel SCC Processor}

The SCC processor [4] is a 48-core experimental processor from Intel, featuring 24 organized into a $4 \times 6$ grid and linked by a $2 \mathrm{D}$ mesh on-chip network. A tile contains a pair of $\mathrm{P} 54 \mathrm{C}$ processor cores, a router, and a $16 \mathrm{~KB}$ block of SRAM, which is used for message passing. Each core has L1 instruction and data caches (16KB each) and a unified level 2 (L2) cache (256KB).

\subsection{Data Flow Process Network Model of Computation}

We assume of a set of concurrently executing deterministic processes, communicating with each other in a point-to-point manner, via bounded FIFOs. 


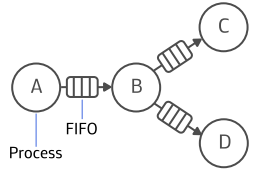

Fig. 1. A Data flow Process Network

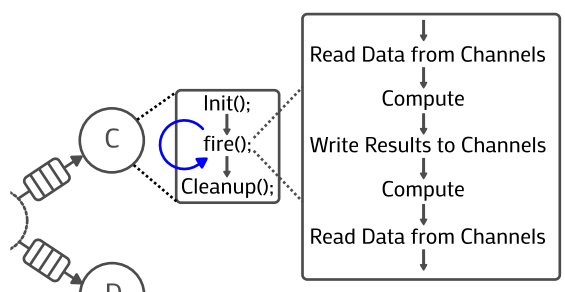

Fig. 2. Structure of a Process

A process attempting to read (write) an empty (full) FIFO blocks (or stalls) till the read (write) operation can be successfully completed A process can only execute (fire) when it has acquired sufficient data to proceed with its computation. Process networks have natural support for parallelism making them an attractive model for describing multiprocessing systems, specially digital signal processing systems.

Structure of the Process. The functionality of each process in a network is split into three parts: initialization, execution and cleanup, with each part executed by a call to a pre-defined function, see Figure 2. The initialization function (init) prepares a process for execution, by initializing variables, and allocating memory, if necessary. The main functionality of the process is encapsulated in the fire function, and runs as long as necessary, continuously reading data from input channels, computing, and writing results to one or more output channels. The fire function therefore, consists of compute segments, surrounded by channel read and/or channel write segments. The compute segments involve purely processing of already acquired data, and the time to execute each of these segments depends on the nature of acquired data. The segment with the longest computing time is called the dominating segment. The cleanup function is called once the process terminates its core functionality, and is used for returning resources to the system, or gathering performance statistics.

The Master Process and the Modified Process Structure. Controlling the behaviour of the application at runtime is achieved via a special process, called the master process, which can send appropriate control messages to the application at runtime. For brevity, we only discuss the stop control message, which is used to indicate to a process that it must exit its fire function and proceed to execute the cleanup function. Its structure follows the layout of the process in Figure 2.

Further, in order that a process can react to control messages, its structure has been modified as shown in Figure 4. After every call to Read (or Write) function, the process checks for the control message (for example, stop), and takes appropriate action. Inserting a check for control message after every call to Read or Write is automatically done at the pre-compilation stage. In the 


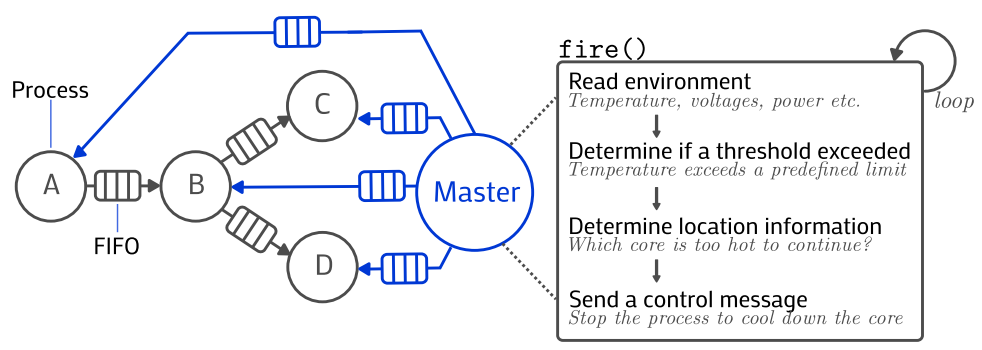

Fig. 3. An example of the master process. Additional control channels are also shown

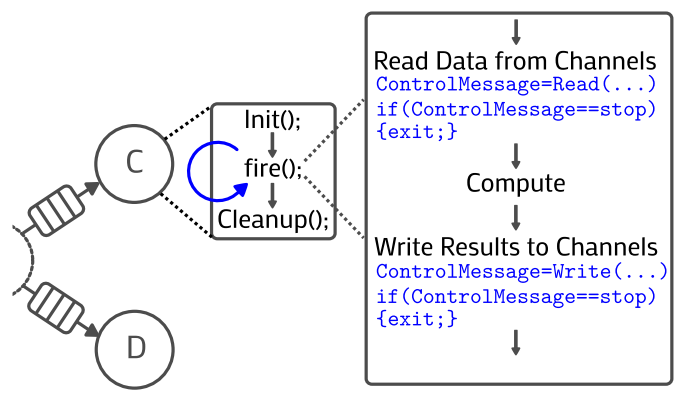

Fig. 4. Modified process structure

rest of this paper, the process from the original network is denoted simply as a process in order to distinguish it from the master process.

\section{Achieving Predictable Timing Characteristics}

\subsection{Definitions}

For the purpose of this paper, predictable timing characteristics consists of three parts: (a) Observed time to execute any given compute segment is within a known bound; (b) Observed time to send a fixed size message is within a known bound; and (c) Observed time for an application to react to a control message is within a known bound.

We define reaction time of a process as:

$$
R_{p}=t_{p, C^{*}}-t_{m, C^{*}}
$$

where $R_{p}$ is the reaction time of a process $p, t_{p, C^{*}}$ is the time when a process receives the control message, and $t_{m, C^{*}}$ is the time when the master queues a control message for transmission (i.e., when a call to iRCCE/RCCE_send is placed).

\subsection{Proposed Solution}

The proposed solution has been divided into the following sections: 
1. A new communication interface such that for any process, the delay in receiving a control message is independent of the size of data messages being exchanged between processes, thus ensuring bounded reaction times;

2. Mapping between processes and cores which reduces uncertainties in communication times between processes;

3. Eliminating factors that may lead to unpredictable compute segment times.

\subsection{A New Communication Interface}

We do not place any limit on the length of data messages exchanged between processes. However, arbitrarily long messages lead to arbitrarily long communication times, leading to unpredictable delays in reading a control message from the master. Clearly, in order to ensure bounded reaction times, the control message communication must be decoupled from the normal data communication. In addition, as per the assumed model of computation, it must be ensured that a process attempting to read a channel with insufficient data must not proceed further as long as all data has not been acquired. Therefore, we propose a Read function, which is called by the process to acquire data, and is implemented on top the iRCCE library:

$$
\text { ControlMessage }=\operatorname{Read}(\text { ID, } * \text { buffer, size, source); }
$$

The function returns Controlmessage, holding the value of any control message, if received, and all other symbols have usual meanings. In order to decouple data messages from control messages, the Read function:

1. Receives an arbitrary sized message in chunks not exceeding a fixed size;

2. Intersperses checking for a control message from the master with reading data messages;

As indicated earlier, if a process receives a control message (e.g. stop), the process exits its fire function and proceeds to cleanup, see Figure 4 . In order to ensure that the MPB has guaranteed space for holding control messages, we require that:

$$
\text { CHUNK_SIZE }+\left|C^{*}\right| \leq|M P B|
$$

Where $\left|C^{*}\right|$ is the maximum size of the control message, and $|M P B|$ is the size of the MPB on each tile of the Intel SCC. Very small values of chunk size results in a large number of calls to the communication library, leading to inefficiency, but also decrease the latency with which a process checks for a control message. Thus, the choice chunk size reflects a tradeoff made by the designer at compile time. It is clear that the sender process must co-operate with the receiver process, and therefore, it must split a large message in small chunks, each not exceeding CHUNK_SIZE in size. The above discussion is summarized in Figure 5. Referring to Figure 5, the receiver process expects $\mathrm{n}$ bytes from the sender, and therefore calls its Read function. The communication interface reads the message in chunks, interleaving with reading (if any) control message from

\footnotetext{
${ }^{1}$ The discussion for the corresponding Write function is analogous.
} 


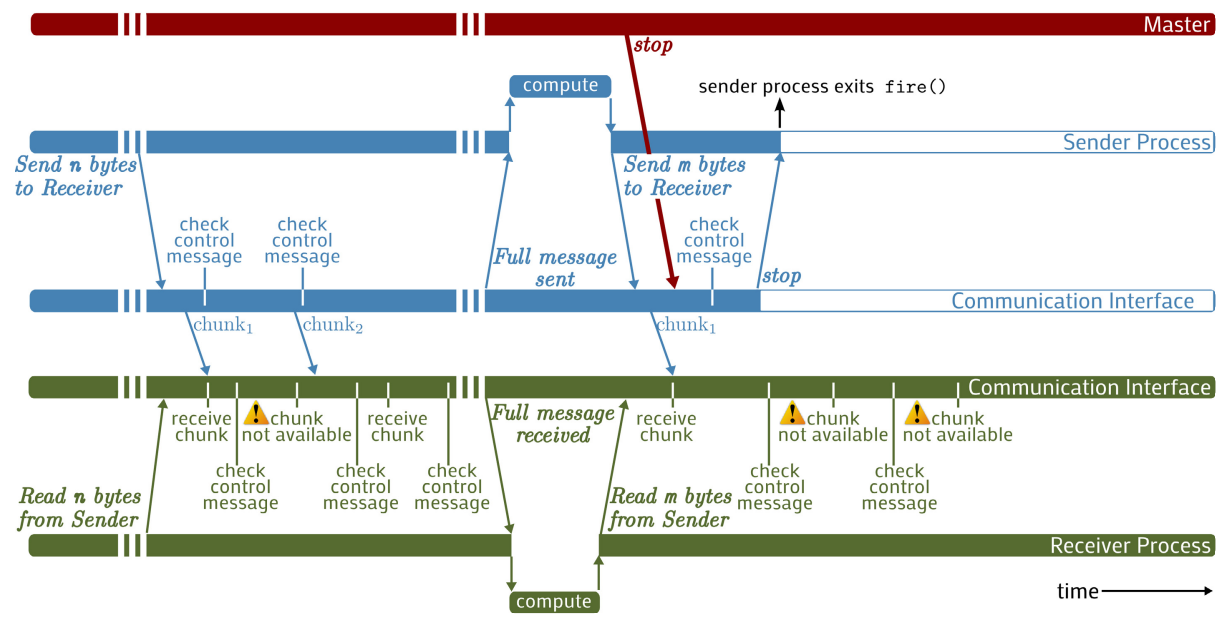

Fig. 5. Timeline of two communicating processes along with a master

the master. Notice that all processes (including the master) are asynchronous, and there may be instants when the receiver expects to receive a data chunk from the sender, but is not successful. In such situations, the communication interface must not block, and must proceed to checking for the control messages from the master. In order to ensure that the communication interface does not return to the caller without having acquired sufficient data, the communication interface keeps a running count of total message size received with each new chunk. Such non-blocking receive for data messages justifies the use of nonblocking communication library, such as the iRCCE.

Timing Bounds The worst case reaction time of a process is defined as follows:

$$
R_{p}^{*}=t_{\text {read } \mid \text { write }}^{*}+S_{p}^{*}+t_{m, C^{*}}^{*}
$$

where $R_{p}^{*}$ is the worst case reaction time of process $\mathrm{p}, t_{\text {read } \mid \text { write }}^{*}$ is the maximum time to completely receive (or send) one CHUNK_SIZE of message, $S_{p}^{*}$ is the length of dominating segment of process $\mathrm{p}$, and $t_{m, C^{*}}^{*}$ is the maximum time to transport the control message from the master to process $\mathrm{p}$.

\subsection{Process-to-Core Mapping}

The mapping of processes to cores impacts timing properties of an application due to at least two factors:

Process Interference : Unless carefully scheduled, two or more processes sharing the same core lead to non-deterministic demands on the available computing resources (such as MPB, tile router), thereby causing non-real time behaviour. The timing performance of the application is improved by mapping only one process to a tile (and switching off the unused core). 
Communication Traffic Interference : If message streams between more than one pair of sender-receiver processes contend at an intermediate router, then, timing disturbance due to such cross traffic may be observed at both pairs of processes. Therefore, processes must be mapped in a manner that minimizes cross traffic at all active routers on the chip, see [10.

\subsection{Eliminating Other Interferences}

Timing predictability of an application is further improved by disabling all interrupts and L2-caches, which is a common practice in the design of real time systems.

\section{Experiments and Results 2}

\subsection{Methodology}

Since reaction time (see (3) ) is influenced by dominating segments as well as communication, the overall objective of this section is to show that $R^{*}$ computed using (3) upper bounds all values of reaction times observed when running real applications. In order to determine representative values for $t_{\text {read } \mid \text { write }}^{*}, S_{p}^{*}$ and $t_{m, C^{*}}^{*}$, a series of calibration experiments are performed on the Intel SCC:

Message Read-Write Timings. A simple producer process was mapped to core 0 and a simple consumer process was mapped at hop distances of 1,2,3 and 4 from the producer. The message size transmitted by the producer ranged from 32 bytes to $3 \mathrm{~KB}$. Timings were recorded under ideal and noisy conditions, and were measured with reference to the core's local time-stamp counter (TSC). In order to ensure that the reported timings are correct, the sender and receiver clocks were first synchronized using a barrier, and then, the sender transmitted its local time stamp to the receiver. The receiver computed the message travel time by referencing the received time-stamp with its own on-core time stamp counter, and thereby avoiding costly accesses to the global time-stamp counter. In the first set of experiments, only the producer-consumer pair was active on the chip, and the observed timing model is summarized in (44). The reported model matches well with the timings reported in [6].

$$
t_{p \rightarrow p^{\prime}}(\text { clock cycles })=5.289|P|+57.53|H|+894.4
$$

Where $t_{p \rightarrow p^{\prime}}^{*}$ is the worst-case time in delivery a given message, $|P|$ is the packet size of the message in bytes, and $|H|$ is the hop-distance between the sender and the receiver (measured along the message transport route). Next, one traffic generator process was mapped onto one core in each tile of the SCC, with the objective of creating heavy traffic at each router of the SCC. The producer and consumer processes did not shared any core with the traffic generators. The corresponding worst case time is presented in (15).

\footnotetext{
${ }^{2}$ Experiments were performed on a remote baremetal SCC.
} 


$$
t_{p \rightarrow p^{\prime}}^{*}(\text { clock cycles })=4.908|P|+9921
$$

Notice that the worst case time is independent of the hop distance between cores. Each model was derived out of a total of 800 observations. Note that the reported times include both the message transmission time, and the time to read the full message by the consumer.

Dominating Segments. Dominating segments were evaluated by carefully isolating computing segments from communicating segments in each process of the selected application. Next, the application was run with an input known to lead to high compute times. For signal processing application, these inputs are known in advance. For instance, for Demosaicing, a RAW image with lot of high-frequency components will lead to high compute times. From the calibration runs, the dominating segment of each process is calculated.

Once all calibration runs are complete, the upper bound on reaction times $\left(R^{*}\right)$ is calculated using (3).

\subsection{Results}

For experiments, two large applications, the Demosaicing [5], and the motionJPEG (MJPEG) decoder are selected. The SCC was booted with the following parameters: tile clock: $533 \mathrm{MHz}$, router: $800 \mathrm{MHz}$, DDR3: $800 \mathrm{MHz}$, with L2-caches disabled. The size of control messages was set to 32 bytes, and CHUNK_SIZE was set to $3 \mathrm{~KB}$. Both applications are compute and communication intensive.

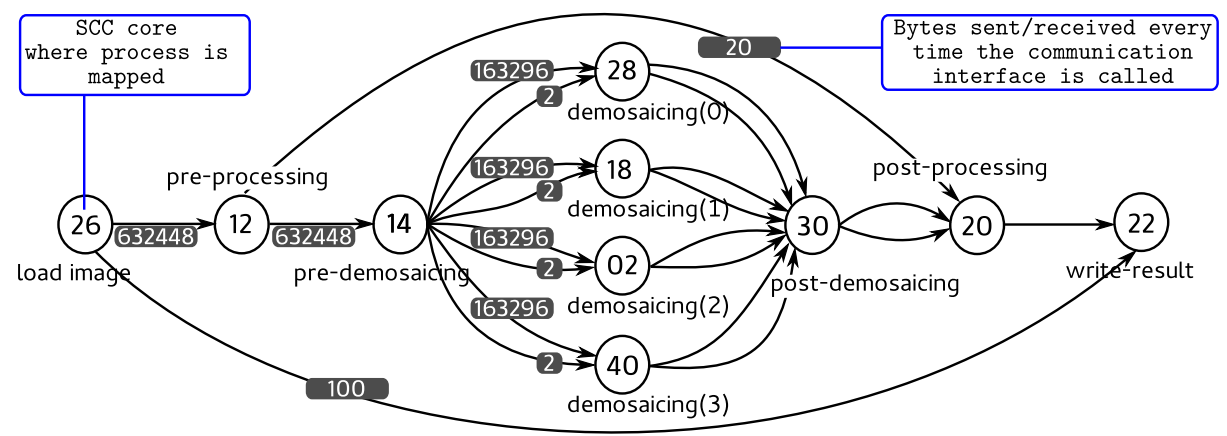

Fig. 6. The Demosaicing application with the mapping details. Size of data messages exchanged between processes is also shown. Master process was mapped to core 24 .

Reaction Times for the Demosaicing Application. The Demosaicing process network is shown in Figure 6. Notice that the process-to-core mapping ensures that one tile does not host more than one process, and the cross traffic at routers is minimised. Reaction times for each process in the Demosaicing application are shown in Figure 8, with lines representing observed values from experiments, while the bars represent the bounds computed during calibration runs. Notice that the bounds are tight. 


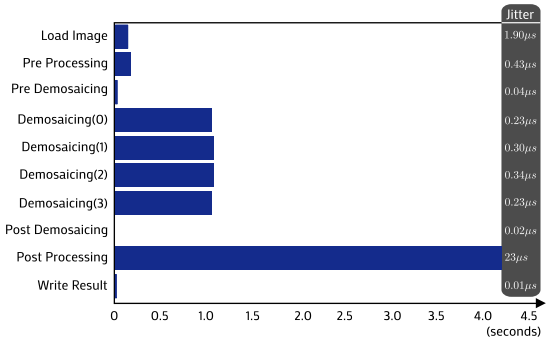

Fig. 7. Lengths of dominating segments for each process in the Demosaicing application

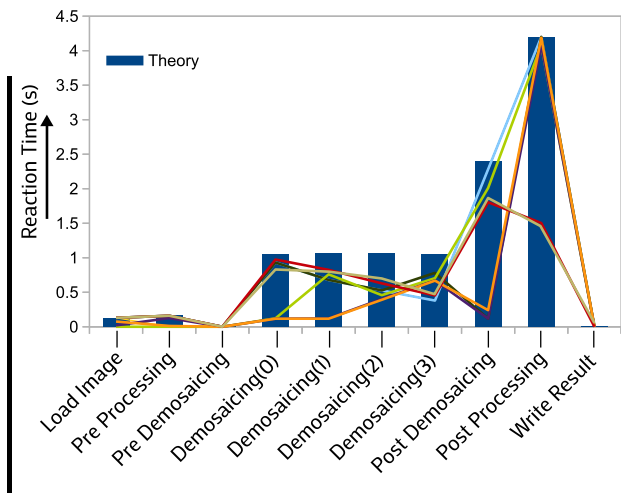

Fig. 8. Reaction Times for the Demosaicing application for 10 observations. Lines show observed timings during experiments.

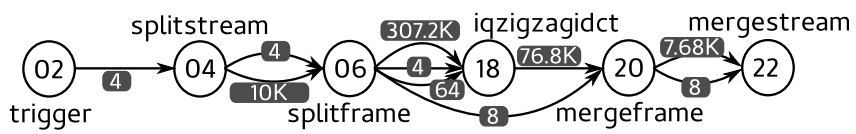

Fig. 9. The Motion JPEG decoder. Master process was mapped to core 16.

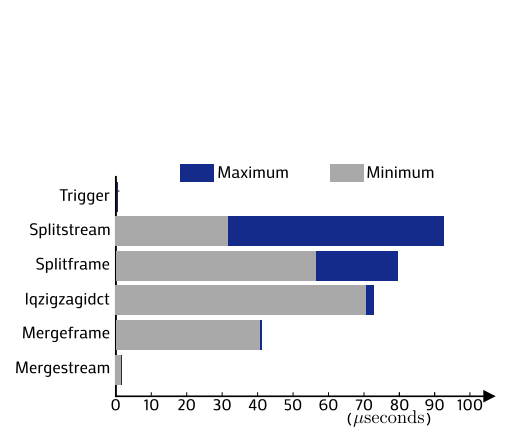

Fig. 10. Size of dominating segments for the MJPEG decoder

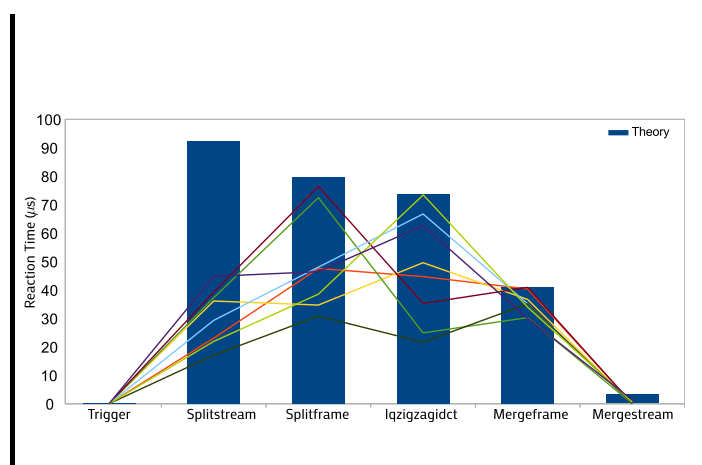

Fig. 11. Reaction Times for the MJPEG decoder for 10 observations

Reaction Times for the Motion-JPEG Decoder. The MJPEG decoder process network is shown in Figure 9. The splitstream and splitframe processes search for certain markers in the encoded image. The amount of time spent in searching for the marker depends on the frame itself, and hence, the dominating segments in these processes show large variations. Consequently, for calculating worst case reaction time $R_{p}^{*}$, the maximum size of corresponding dominating segments is used. Figure 10 shows the lengths of dominating segments for the MJPEG application, while Figure 11 compares the predicted value of reaction times to those observed over 10 experiments involving 30 frames each. Notice that unlike the 
Demosaicing application, reaction times in MJPEG application are not determined solely by the dominating segments, but are also influenced by communication times. Also notice that the iqzigzagidct process communicates messages of size $76.8 \mathrm{~KB}$, which according to (5) would take $725 \mu \mathrm{s}$. Unless the entire message was communicated in chunks, the reaction times would have been an order of magnitude more than what is reported in Figure 11.

\subsection{Overhead}

The definition of the new communication interface was common to both applications, and the additional logic for chunk-based message communication resulted in a mere 528 byte overhead. The chunk-based message communication improved the timing efficiency of message communication, leading to a $4.7 \%$ improvement in per-frame timing for MJPEG decoder. This is because chunk-based communication mitigated the number of times shared memory was accessed during message passing, which more than compensated for larger number rounds required to send the complete message. Any time overhead for the Demosaicing application was negligible since timings in this case are determined by dominating segments.

\section{Summary}

We demonstrated that it is indeed possible to achieve predictable runtime performance, and bounded reaction time of applications on the Intel SCC processor. To this end, we proposed a non-blocking communication strategy which interleaves data and control communication, and also complies with the execution semantics of general data flow process networks. This strategy enables a process (or an entire process network) to react to events with a bounded and predictable latency. Furthermore, the above-mentioned strategy was encapsulated in an RCCE- (or MPI-) like communication API, making it possible to easily incorporate the proposed strategy into existing multi- and many-core application design frameworks. Experiments involving two representative applications validate our technique and show that predictable performance, including bounded reaction latencies can be achieved easily on baremetal SCC, and indeed on any many-core processor.

Acknowledgements. This work was supported by EU FP7 project EURETILE, under grant numbers 247846. Lars Schor was also partially supported by an Intel PhD Fellowship.

\section{References}

1. http://www.kalray.eu/

2. http://www.tilera.com/products/processors

3. Clauss, C., et al.: Evaluation and Improvements of Programming Models for the Intel SCC Many-Core Processor. In: Proc. HPCS, pp. 525-532 (2011) 
4. Howard, J., et al.: A 48-Core IA-32 Message-Passing Processor with DVFS in 45nm CMOS. In: Proc. ISSCC, pp. 108-109 (2010)

5. Li, X.: Demosaicing by successive approximation. IEEE Transactions on Image Processing 14(3), 370-379 (2005)

6. Mattson, T.G.: Using Intel's Single-Chip Cloud Computer (SCC), http://communities.intel.com/docs/DOC-19269 (online February 2012)

7. Mattson, T.G., et al.: The 48-core SCC Processor: The Programmer's View. In: Proc. SC, pp. 1-11 (2010)

8. Puffitsch, W., Noulard, E., Pagetti, C.: Mapping a multi-rate synchronous language to a many-core processor. In: 2013 IEEE 19th Real-Time and Embedded Technology and Applications Symposium (RTAS), pp. 293-302 (2013)

9. van der Wijngaart, R.F., et al.: Light-weight Communications on Intel's Single-chip Cloud Computer Processor. SIGOPS Oper. Syst. Rev. 45(1), 73-83 (2011)

10. Zimmer, C., Mueller, F.: Low contention mapping of real-time tasks onto tilepro 64 core processors. In: 2012 IEEE 18th Real-Time and Embedded Technology and Applications Symposium (RTAS), pp. 131-140 (2012)

11. Ziwisky, M., et al.: BareMichael: A Minimalistic Bare-Metal Framework for the Intel SCC. In: Proc. MARC, pp. 66-71 (2012)

12. Åkerholm, M., Samuelsson, T.: Design and Benchmarking of Real-Time Multiprocessor Operating System Kernels. Master's thesis, Mälardalen University (2002) 